\title{
A!
}

This is an electronic reprint of the original article.

This reprint may differ from the original in pagination and typographic detail.

Rodriguez, M.; Paraoanu, G. S.; Törmä, P.

\section{Vortices in trapped superfluid fermi gases}

Published in:

Physical Review Letters

DOI:

10.1103/PhysRevLett.87.100402

Published: 03/09/2001

Document Version

Publisher's PDF, also known as Version of record

Please cite the original version:

Rodriguez, M., Paraoanu, G. S., \& Törmä, P. (2001). Vortices in trapped superfluid fermi gases. Physical Review Letters, 87(10), 1-4. [100402]. https://doi.org/10.1103/PhysRevLett.87.100402

This material is protected by copyright and other intellectual property rights, and duplication or sale of all or part of any of the repository collections is not permitted, except that material may be duplicated by you for your research use or educational purposes in electronic or print form. You must obtain permission for any other use. Electronic or print copies may not be offered, whether for sale or otherwise to anyone who is not an authorised user. 


\title{
Vortices in Trapped Superfluid Fermi Gases
}

\author{
M. Rodriguez, ${ }^{1}$ G.-S. Paraoanu, ${ }^{2}$ and P. Törmä ${ }^{1}$ \\ ${ }^{1}$ Laboratory of Computational Engineering, P.O. Box 9400, FIN-02015 Helsinki University of Technology, Finland \\ ${ }^{2}$ Department of Physics, Loomis Laboratory, $1110 \mathrm{~W}$. Green Street, University of Illinois at Urbana-Champaign, \\ Urbana, Illinois 61801
}

(Received 25 April 2001; published 20 August 2001)

\begin{abstract}
We consider a superfluid of trapped fermionic atoms and study the single vortex solution in the Ginzburg-Landau regime. We define simple analytical estimates for the main characteristics of the system, such as the vortex core size, temperature regimes for the existence of a vortex, and the effects of rotation and interactions with normal fermions. The parameter dependence of the vortex core size (healing length) is found to be essentially different from that of the healing length in metallic superconductors or in trapped atomic Bose-Einstein condensation in the Thomas-Fermi limit. This is an indication of the importance of the confining geometry for the properties of fermionic superfluids.
\end{abstract}

DOI: $10.1103 /$ PhysRevLett.87.100402

Experiments on cooling of trapped gases of Fermionic alkali-metal atoms $[1-4]$ are at the level where the achievement of the predicted BCS transition [5,6] can be anticipated. Cooper-paired trapped atoms allow us to study and test the BCS theory in a controlled manner-for instance the classic problem of the BCS-BEC (Bose-Einstein condensation) crossover when the interparticle attraction varies [7] could be studied using the possibility to tune the interatomic scattering length. Several methods for observing the existence of a gap in the excitation spectrum of the superfluid Fermi gas have been proposed $[8,9]$.

Vortices are a macroscopic signature of the superfluidity, and the vortex core size reflects the typical coherence lengths of the system. Vortices have played a major role in experimental and theoretical studies of superfluid helium [10] and lately, of condensates of Bosonic atoms [11]. We consider vortex solutions for superfluid trapped Fermi gases. Fermionic alkali gases are dilute and weakly interacting; moreover, the origin of superfluidity is $s$-wave (singlet) pairing between atoms in two different hyperfine states, whereas $p$-wave (triplet) scattering is negligible $[6,12]$. Thus the system is very different from superfluid ${ }^{3} \mathrm{He}$ and can be described by simple BCS theory with a single complex-number order parameter.

Although a full description of the system would require a careful investigation of the Bogoliubov-de Gennes (BdG) equations $[13,14]$, we have chosen as a first attempt to characterize the system using the Ginzburg-Landau (GL) equation in a trapped geometry [15]. This allows us to define intuitive estimates for the vortex core size and for the temperature regimes where a vortex solution exists. Furthermore, the effects of rotation as well as of interactions with normal fermions [position-dependent Hartree fields (HF)] can be incorporated into the formalism. We estimate their effects on the critical temperature and the vortex solution, and develop a self-consistent method for treating the effect of the Hartree fields. We also discuss how our results can be used in estimating the possibilities for observing a vortex.
PACS numbers: 05.30.Fk, 32.80.-t, 74.25.-q

The GL equation for atoms in two different hyperfine states confined in a symmetric trap of frequency $\Omega$ is given by [15]

$$
\begin{array}{r}
{\left[-\frac{7 \zeta(3)}{48 \pi^{2}}\left(\frac{\hbar \Omega}{k_{B} T}\right)^{2} \nabla^{2}+\frac{1+2 \lambda}{2 \lambda} R^{2}-\ln \frac{T_{c}^{(0)}}{T}\right] \Delta(R)} \\
+\frac{7 \zeta(3)}{8 \pi^{2}} \frac{|\Delta(R)|^{2}}{\left(k_{B} T\right)^{2}} \Delta(R)=0,
\end{array}
$$

where $\lambda=2 p_{F}^{(0)}|a| /(\hbar \pi)$ is the interaction parameter $\left(<1\right.$ for dilute systems), $T_{c}^{(0)}$ is the critical temperature for the corresponding homogeneous system, $\Delta\left(R=\mathbf{r} / R_{\mathrm{TF}}\right)$ is the spatially dependent order parameter, and $R_{\mathrm{TF}}$ is the cloud size $R_{\mathrm{TF}}=\left[2 E_{F} /\left(m \Omega^{2}\right)\right]^{1 / 2}$ given by the Thomas-Fermi (TF) approximation [16].

We impose a singly quantized vortex solution for the order parameter in cylindrical coordinates in a general anharmonic trap potential: $\Delta(R)=\Delta(\rho, z, \theta)=e^{i \theta} \Delta(\rho, z)$ where $\rho \equiv \frac{\rho}{R_{\mathrm{TF}}}, z \equiv \frac{z}{R_{\mathrm{TF}}}$, and the equation for the real order parameter becomes (in $\hbar=1$ units)

$$
\begin{gathered}
{\left[-\frac{1}{2 m^{*}}\left(\frac{\partial^{2}}{\partial \rho^{2}}+\frac{1}{\rho} \frac{\partial}{\partial \rho}+\frac{\partial^{2}}{\partial z^{2}}-\frac{1}{\rho^{2}}\right)\right.} \\
\left.+\frac{m^{*} \omega^{* 2}}{2}\left(\rho^{2}+A z^{2}\right)-E_{\mu}(T)\right] \Delta(\rho, z) \\
+C(T)|\Delta(\rho, z)|^{2} \Delta(\rho, z)=0, \\
m^{*}=\frac{1}{2} \frac{48 \pi^{2}}{7 \zeta(3)}\left(\frac{k_{B} T_{c}}{\Omega_{\mathrm{ho}}}\right)^{2}\left(\frac{T}{T_{c}}\right)^{2} \\
\omega^{*}=\sqrt{\frac{1+2 \lambda}{\lambda m^{*}}}, \quad C=\frac{7 \zeta(3)}{8 \pi^{2}}\left(\frac{T_{c}}{T}\right)^{2} \\
E_{\mu}(T)=\ln \left(T_{c}^{(0)} / T\right)=\ln \left(T_{c} / T\right)+3 \tilde{\omega} / 2
\end{gathered}
$$

and $\frac{3}{2} \tilde{\omega}=\ln \frac{T_{c}^{(0)}}{T_{c}}$. We use the scaling $\Delta(R) \equiv \Delta(R) /$ $\left(k_{B} T_{c}\right)$. The asymmetry of the trapping potential is described by $A=\frac{\Omega_{\perp}^{2}}{\Omega_{z}^{2}}$, and $\Omega_{\mathrm{ho}}=\left(\Omega_{\perp}^{2} \Omega_{z}\right)^{1 / 3}$. Thus the GL

(C) 2001 The American Physical Society

100402-1 
equation becomes analogous to the Gross-Pitaevskii (GP) equation for bosonic atoms [11] for particles of an effective mass $m^{*}$ in a harmonic confining potential with an effective frequency $\omega^{*}$ and $\omega_{z}^{*}=\omega^{*} \sqrt{A}$. The nonlinear term plays the role of repulsive interparticle interaction, and $E_{\mu}(T)$ is the "chemical potential" of the system. Strong superfluidity, compared to trap energy scales, means large effective mass and small effective trapping frequency.

We consider a two-dimensional geometry where the single vortex is uniform along its axis ( $z$ axis). Equation (3) then reads

$$
\begin{aligned}
& {\left[\frac{1}{2 m^{*}}\left(\frac{\partial^{2}}{\partial \rho^{2}}+\frac{1}{\rho} \frac{\partial}{\partial \rho}-\frac{1}{\rho^{2}}\right)+\frac{m^{*}\left(\omega^{*}\right)^{2}}{2} \rho^{2}-\right.} \\
&\left.E_{\mu}(T)\right] \Delta(\rho)+C(T)|\Delta(\rho)|^{2} \Delta(\rho)=0,
\end{aligned}
$$

and now $E_{\mu}(T) \equiv \ln \left(T_{c} / T\right)+\tilde{\omega}$. We have used the steepest descent method described, e.g., in [17] using imaginary time propagation to search for the ground state solution of Eq. (6). We applied the Crank-Nicholson finite differencing method used to solve the GP equation, but without normalization after each step as the size of $\Delta$ is determined by the nonlinear term.

Vortex core sizes. - The size of the vortex core reflects the healing length of a superfluid because within this distance, the order parameter "heals" from zero up to its bulk value. The first guess to estimate the healing length in our case would be to equate the "kinetic energy" term in Eq. (6), $\sim 1 /\left(2 m^{*} \xi^{2}\right)$ to the "interaction energy," as was done in the case of trapped atomic BEC (GP equation) [18]. This yields ( $\xi$ is in $R_{\mathrm{TF}}$ units)

$$
\xi^{2}=1 /\left[2 m^{*} C(T)|\Delta|^{2}\right]
$$

where $|\Delta|^{2}$ is the "density" of Cooper pairs (in $k_{B} T_{c}$ units). In the case of the TF approximation for BEC, the order parameter is substituted by its value in the middle of the trap. Making a corresponding substitution here gives $\xi^{2}=\frac{1}{2 m^{*} E_{\mu}(T)}$ which is the same as the definition of the correlation length in metallic superconductors in the GL regime. However, it does not correctly describe the numerically obtained values for the healing length because, for the experimentally feasible parameters used here, the energy scales in the GL equation do not correspond to the TF limit of BEC.

We propose a measure for the vortex core size by demanding that the kinetic plus potential energy term in Eq. (6) has its minimum value. This term corresponds to a vortex in a harmonic trap, that is, the first excited state. The energetically favored position is thus the maximum of the first excited state wave function which coincides with the oscillator ground state length. We thus define

$$
\xi^{2}=\frac{1}{m^{*} \omega^{*}}=\sqrt{\frac{7 \zeta(3)}{24 \pi^{2}}}\left(\frac{T_{c}}{T}\right)\left(\frac{\Omega}{k_{B} T_{c}}\right) \sqrt{\frac{\lambda}{2 \lambda+1}} .
$$

Figure 1 shows the order parameter for a selected set of parameters, together with the two estimates, Eqs. (7) and (8) for the healing length. The results fit excellently with Eq. (8), whereas the deviation from Eq. (7) is considerable and qualitatively different for small and large $\Delta$. The definition of Eq. (8) is a function of temperature decreasing as $T$ approaches $T_{c}$ (we have confirmed this temperature dependence also numerically). This is an opposite behavior to that of the GL healing length $\xi_{\mathrm{GL}} \sim 1 / \sqrt{1-T / T_{c}}$ appearing in studies of metallic superconductors and helium. The trapping energy becomes relatively stronger as $T \rightarrow T_{c}$ because $\Delta$ decreases. This means stronger confinement for $\Delta(\rho)$ and decreasing $\xi$. Note that also in BEC, the confinement determines the healing length if one is away from the TF regime [19]. Differences to BEC arise, however, from the temperature dependence of the GL equation and from the normalization.

Critical temperature for a trapped system. - As pointed out in [15] one can use the GL equation to estimate the critical temperature of the trapped system, as compared to the corresponding homogeneous case. Close to $T_{c}$ the nonlinear term is negligible and the GL equation reduces to the Schrödinger equation for a trapped particle, $E_{\mu}(T)$ now denoting the energy. The smallest possible energy $E_{\mu}\left(T_{c}\right)=\frac{3}{2} \tilde{\omega}$ is then simply the ground state energy of the trap, $\omega^{*}+\frac{1}{2} \omega_{z}^{*}$. Equating $\frac{3}{2} \tilde{\omega}=\omega^{*}+\frac{1}{2} \omega_{z}^{*}$ gives $T_{c}$ in terms of $T_{c}^{(0)}, \omega^{*}$ and $\omega_{z}^{*}$ by the definition $\tilde{\omega}=$ $\frac{2}{3} \ln \left(T_{c}^{(0)} / T_{c}\right)$. We use this to calculate the new critical

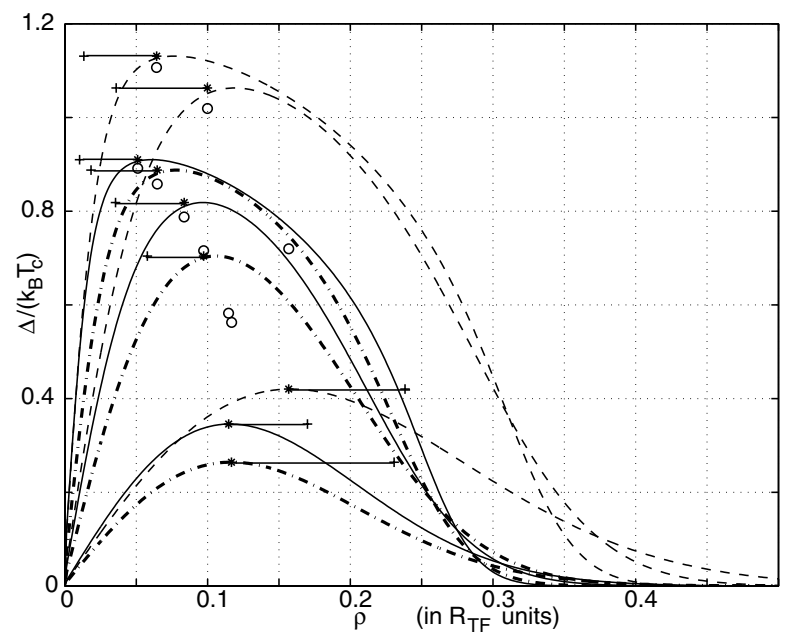

FIG. 1. Vortex solutions for the order parameter in $k_{B} T_{c}$ units and in trap units. Solid lines are for $\Omega=2 \pi \times 100 \mathrm{~Hz}, 1 \mathrm{kHz}$, and $3 \mathrm{kHz}\left(N=3 \times 10^{5},|a|=1140 \AA\right.$, and $\left.T=0.89 T_{c}\right)$. Dashed lines correspond to $N=10^{5}, 3 \times 10^{5}, 10^{6}\left(T=0.8 T_{c}\right.$, $\Omega=820 \mathrm{~Hz}$, and $|a|=1140 \AA$ ). Dot-dashed lines are for $|a|=985,1118$, and $1608 \AA\left(N=3 \times 10^{5}, \Omega=820 \mathrm{~Hz}\right.$, and $T=0.89 T_{c}$ ). In all three cases, the highest value of $\Omega, N$, or $|a|$ corresponds to the curve with the largest maximum $\Delta$. The healing lengths given by Eqs. (8) and (7) are represented by $*$ and + , respectively. The estimate for $\Delta_{\max }$ given by Eq. (11) is represented by $\bigcirc$. 
temperatures when the effects of rotation and Hartree fields are added. Below we estimate the temperature regimes for vortex solutions using a similar argument.

Temperature regimes for the existence of a vortex solution.-The ground state energy of the trapped superfluid in the quasilinear regime can be estimated as above to be $E_{\mu}\left(T_{c}\right)=\omega^{*}$ (in 2D). A vortex has a higher energy, and $E_{\mu}(T)=\ln \left(T_{c} / T\right)+\omega^{*}$ must have a large enough value in order for the GL equation to have a solution. The minimum extra energy that a vortex in a trapped noninteracting superfluid requires is the $2 \mathrm{D}$ harmonic oscillator energy $\omega^{*}$ (this is also the vortex energy for a noninteracting BEC [20]). Thus the maximum temperature $T_{v}$ at which $E_{\mu}(T)$ can provide this extra energy is given by $\ln \left(T_{c} / T_{v}\right)=\omega^{*}$. We have checked the validity of this estimate for a system of $N=3 \times 10^{5}$ atoms, scattering length $|a|=1140 \AA$, and trap frequency $\Omega=820 \mathrm{~Hz}$. The maximum $T$ at which a vortex solution can exist, estimated by $T_{c}=T_{v} e^{\omega^{*}}=T_{v} e^{0.0146\left(T_{c} / T_{v}\right)}$, is $T_{v} \sim 0.98 T_{c}$. The actual maximum $T$ where the GL equation gives a numerical solution was about $0.97 T_{c}$. The deviation is due to neglecting the nonlinear term, and $T_{v}$ can be understood as the upper bound for the maximum temperature.

Hartree fields and rotation. - In practical systems, the Cooper-paired atoms are always interacting with the normal part of the gas whose density distribution is now position dependent. Moreover, the whole system may be rotating, cf. vortices in atomic BEC [11]. The GL equation used in this paper was derived in [15] using the TF approximation [16] for the density profile of the trapped Fermi gas in a harmonic symmetric potential. We follow this derivation and add a new potential term $V(\mathbf{r})$ in the local density approximation. This potential can describe, e.g., the Hartree fields or rotation. It is assumed to be small enough in the sense that one can still use the quasiclassical expression for the product of two Green's functions in Eq. (7) of [15]. We obtain the GL solution as given by Eq. (9) of [15] but with a new term in the expansion of the Fermi energy around $R=0$ (see also [21]) given by $\varepsilon_{F}(R) / \varepsilon_{F}=1-R^{2}-V(R)$, where $R=\mathbf{r} / R_{\mathrm{TF}}$ and the $R_{\mathrm{TF}}=v_{F} / \Omega$. Note that $R_{\mathrm{TF}}$ and $\Omega$ might have changed because of adding $V(\mathbf{r})$. Expanding to second order and derivating with respect to $\Delta^{*}$ the "potential term" in the GL equation now reads

$$
\frac{1+2 \lambda}{2 \lambda}\left[R^{2}+V^{(2)}(R)\right]
$$

where $V^{(2)}(R)$ denotes an expansion of $V$ to second order. The potential $V(R)$ has to be smooth enough for a second order expansion to be sufficient.

Effect of rotation on the critical temperature and the healing length.-The TF approximation with a rotation term $V(\rho)=-\omega L_{z}=-\frac{1}{2} m \omega^{2} \rho^{2}$ gives $\varepsilon_{F}(R) / \varepsilon_{F}=$ $1-\rho^{2}-(z \gamma)^{2}$ in the rotating frame of reference, where now $R_{\mathrm{TF}}=v_{F} / \sqrt{\left(\Omega^{2}-\omega^{2}\right)}$ and $\gamma=\left[A \Omega^{2} /\right.$ $\left.\left(\Omega^{2}-\omega^{2}\right)\right]^{1 / 2}$, and $A$ is the asymmetry in the trapping potential as defined earlier. This means that both $m^{*}$ and $\omega^{*}$ change. As the trapping frequency of the atoms is smaller now, $\Omega_{\mathrm{ho}}^{r}=\left[\left(\Omega^{2}-\omega^{2}\right) \Omega\right]^{1 / 3}$, the Fermi energy and $\lambda$ decrease: we define $\lambda^{r}=\lambda\left[1-\left(\frac{\omega}{\Omega}\right)^{2}\right]^{1 / 6}$. This gives the new homogeneous system critical temperature $T_{c}^{r(0)}$, and using the same kind of procedure as above for a nonrotating system, the new healing length $\xi_{r}$ and critical temperature $T_{c}^{r}$ can be calculated. In the limit $\left(\frac{\omega}{\Omega}\right)^{2} \rightarrow 1$, the critical temperature $T_{c}^{r(0)} \rightarrow 0$ as for BEC [22] and $\xi^{r}$ as well as $\ln \left(T_{c}^{r(0)} / T_{c}^{r}\right)$ diverge.

Effect of the normal fermions. - The TF approximation including the nonpaired fermions was introduced in [12] giving $\frac{\varepsilon_{F}(R)}{\varepsilon_{F}}=1-R^{2}+\frac{1}{\varepsilon_{F}} \frac{4 \pi \hbar^{2}|a|}{m} n(R)$, where $n(R)$ is the density distribution of the atoms in one hyperfine state. It was shown that the Hartree field increases the critical temperature of the system. This result is also given by our GL treatment: Consider again the weakly nonlinear regime close to $T_{c}$. We have a Schrödinger equation for a spherically symmetric potential but with a new term given by the Hartree field. Because of the smooth shape of the fermionic distribution one can assume $n(R) \sim n^{(2)}(R)$ and use $V^{(2)}(R) \simeq-\frac{1}{\varepsilon_{F}} \frac{4 \pi \hbar^{2}|a|}{m} n(R)$ in Eq. (9). Thus we have effectively a new harmonic symmetric potential $m^{*}\left(\omega^{*}\right)^{2} R^{2} /$ $2-C^{\prime} n(R) \sim-E_{\mathrm{HF}}+m^{*}\left(\omega^{* \mathrm{HF}}\right)^{2} R^{2} / 2$ with $s=$ $\left(\omega^{* \mathrm{HF}} / \omega^{*}\right)^{2}>1$. That is, the potential is now deeper and with higher "frequency." Because of the new higher Fermi energy $\varepsilon_{F}^{\mathrm{HF}}=\varepsilon_{F}+E_{\mathrm{HF}}$ the critical temperature in the corresponding homogeneous system, $T_{c}^{(0) \mathrm{HF}}$, increases. Simple considerations give $\ln \left(T_{c}^{\mathrm{HF}(0)} / T_{c}^{\mathrm{HF}}\right)=$ $\ln \left(T_{c}^{(0)} / T_{c}\right) s^{1 / 2}$. This means that $T_{c}^{\mathrm{HF}}$ actually deviates more from $T_{c}^{\mathrm{HF}(0)}$ than $T_{c}$ from $T_{c}^{(0)}$; the effect of trapping is enhanced because also the normal fermions feel the trapping potential. On the other hand, $T_{c}^{\mathrm{HF}(0)}>T_{c}^{(0)}$, and for the parameter values we have considered the total effect is that the Hartree fields increase the critical temperature $\left(T_{c}^{\mathrm{HF}} \sim 2 T_{c}\right)$.

Self-consistent solution of normal and superfluid fermions.-As a nonrigorous but intuitive first guess towards a self-consistent treatment, we calculate $n(r)$, instead of the TF approximation, by the BCS theory in local density approximation but with $\Delta(r)$ given by the GL equation:

$n(r)=\int \frac{d^{3} k}{(2 \pi)^{3}}\left\{\left|u_{k}(r)\right|^{2} f\left(E_{k}\right)+\left|v_{k}(r)\right|^{2}\left[1-f\left(E_{k}\right)\right]\right\}$,

where $\left|u_{k}(r)\right|^{2},\left|v_{k}(r)\right|^{2}=1 / 2\left[1 \pm \xi_{k} / \sqrt{\xi_{k}^{2}+|\Delta(r)|^{2}}\right]$ and $\xi_{k}(r)=\frac{\hbar^{2} k^{2}}{2 m}-\frac{4 \pi \hbar^{2}|a|}{m} n(r)-\left(\mu-\frac{1}{2} m \Omega^{2} r^{2}\right)$, and $f$ is the Fermi-Dirac distribution. We solve $n(r)$ from this equation, use it in the GL equation, and iterate until sufficient convergence is found. 


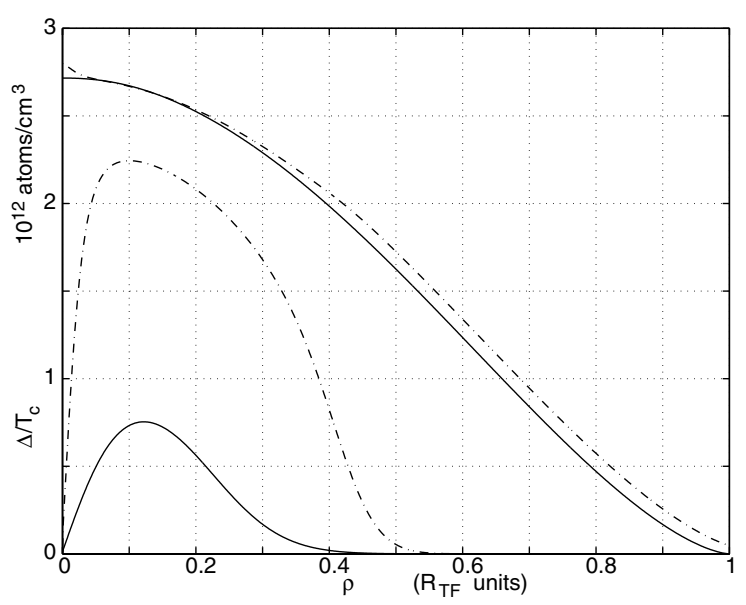

FIG. 2. Vortex solutions for the order parameter are given in $k_{B} T_{c}$ units and density of normal fermions in one hyperfine state in $10^{12}$ atoms $/ \mathrm{cm}^{3}$. Solid lines are the independent initial solutions for Eq. (11) and the GL equation; the dot-dashed lines are the self-consistent solutions. Here $T=0.9 T_{c}, N \sim 3 \times$ $10^{5}, \Omega=820 \mathrm{~Hz}$, and $|a|=1140 \AA$.

The results of the self-consistent calculation are presented in Fig. 2. The order parameter increases considerably, mainly because of the increase in the critical temperature when the Hartree field is added ( $T$ was fixed). Quasiparticles fill the vortex core: there is a small increase of $n(0)$ compared to $n(\xi)$. It would be interesting to compare our simple self-consistent treatment to the rigorous description by the $\mathrm{BdG}$ equations.

Observation of a vortex.-For the parameters used in our calculations, the vortex core sizes/healing lengths vary between $2-10 \mu \mathrm{m}$. This is close to but still above the diffraction limit of light. In principle, for instance, the laser probing method of [9] could be extended to the observation of a vortex: the applied Raman beams are focused so that they intersect either only in the core or only in the superfluid region, and these two choices give absorption peaks at different frequencies.

Also the smallness of the superfluid fraction makes the observation a challenge. In our results, the maximum value of the order parameter (vortex height) is of the same order of magnitude as the temperature. To estimate how it depends on temperature and other parameters, we approximate $\Delta_{\max } \sim \Delta(\rho \sim \xi)$ : we insert $\rho=\xi$ into the GL equation, neglect the first derivative (maximum), and approximate the second derivative by assuming a parabolic shape of $\Delta(\rho)$. This gives (for comparison with the numerics, see Fig. 1)

$$
\Delta_{\max } \sim \sqrt{\left[\ln \left(T_{c} / T\right)-\omega^{*}\right] / C(T)} .
$$

Note that our estimate for the maximum vortex temperature $T_{v}$ corresponds to $\Delta_{\max }$ being real.

We have used the Ginzburg-Landau equation in a trapped geometry to define analytical estimates for the basic quantities describing a trapped superfluid Fermi gas: the vortex core size and height, maximum temperature for a vortex solution, and the changes caused by additional potentials such as rotation or spatially varying Hartree fields. A striking difference to metallic superconductors was found in the temperature and system parameter dependence of the vortex core size/healing length. Our results indicate that the effect of the confining geometry is essential for mesoscopic fermionic superfluids, especially when considering excited state solutions such as vortices.

We thank the Academy of Finland for support (Projects No. 42588, No. 48845, No. 47140, and No. 44897). G.-S.P. also thanks the NSF (Project No. NSF DMR 99-86199).

[1] B. DeMarco and D. S. Jin, Science 285, 1703 (1999); M. J. Holland et al., Phys. Rev. A 61, 053610 (2000).

[2] A. G. Truscott et al., Science 291, 2570 (2001).

[3] M. O. Mewes et al., Phys. Rev. A 61, 011403(R) (2000); see also cond-mat/0011291.

[4] K. M. O'Hara et al., Phys. Rev. Lett. 85, 2092 (2000).

[5] H. T. C. Stoof et al., Phys. Rev. Lett. 76, 10 (1996).

[6] M. Holland et al., cond-mat/0103479.

[7] See M. Randeria, and references therein in Bose-Einstein Condensation, edited by A. Griffin, D. W. Snoke, and S. Stringari (Cambridge University Press, Cambridge, 1995).

[8] W. Zhang et al., Phys. Rev. A 60, 504 (1999); J. Ruostekoski, Phys. Rev. A 60, R1775 (1999); F. Weig and W. Zwerger, Europhys. Lett. 49, 282 (2000); M. A. Baranov and D. S. Petrov, Phys. Rev. A 62, 041601(R) (2000); M. Farine et al., Phys. Rev. A 62, 013608 (2000); G. M. Bruun and C. W. Clark, J. Phys. B 33, 3953 (2000).

[9] P. Törmä and P. Zoller, Phys. Rev. Lett. 85, 487 (2000); G. M. Bruun et al., cond-mat/0011333.

[10] M. M. Salomaa and G. E. Volovik, Rev. Mod. Phys. 59, 533 (1987); R. J. Donelly, Quantized Vortices in Helium II (Cambridge University Press, Cambridge, 1991).

[11] For a review, see, e.g., A. L. Fetter and A. A. Svidzinsky, J. Phys. Condens. Matter 13, R135 (2001), and references therein.

[12] M. Houbiers et al., Phys. Rev. A 56, 4864 (1997).

[13] P. de Gennes, Superconductivity of Metals and Alloys (Addison-Wesley, New York, 1966).

[14] G. M. Bruun et al., Europhys. D 7, 433 (1999); A. Minguzzi et al., cond-mat/0103591.

[15] M. A. Baranov and D. S. Petrov, Phys. Rev. A 58, R801 (1998).

[16] D. A. Butts and D.S. Rokshar, Phys. Rev. A 55, 4346 (1997).

[17] F. Dalfovo and M. Modugno, Phys. Rev. A 61, 023605 (2000).

[18] G. Baym and C. J. Pethick, Phys. Rev. Lett. 76, 6 (1996).

[19] A. L. Fetter, cond-mat/9811366, Chap. II.C.

[20] F. Dalfovo and S. Stringari, Phys. Rev. A 53, 2477 (1996).

[21] M. Houbiers and H. T.C. Stoof, Phys. Rev. A 59, 1556 (1999).

[22] S. Stringari, Phys. Rev. Lett. 82, 4371 (1999). 